\title{
Experimental pain responses in children with chronic pain and in healthy children: How do they differ?
}

\author{
Jennie Cl Tsao PhD, Subhadra Evans PhD, Laura C Seidman BS, Lonnie K Zeltzer MD
}

JCI Tsao, S Evans, LC Seidman, LK Zeltzer. Experimental pain responses in children with chronic pain and in healthy children: How do they differ? Pain Res Manage 2012;17(2):103-109.

BACKGROUND: Extant research comparing laboratory pain responses of children with chronic pain with healthy controls is mixed, with some studies indicating lower pain responsivity for controls and others showing no differences. Few studies have included different pain modalities or assessment protocols.

OBJECTIVES: To compare pain responses among 26 children (18 girls) with chronic pain and matched controls (mean age 14.8 years), to laboratory tasks involving thermal heat, pressure and cold pain. Responses to cold pain were assessed using two different protocols: an initial trial of unspecified duration and a second trial of specified duration.

METHODS: Four trials of pressure pain and of thermal heat pain stimuli, all of unspecified duration, were administered, as well as the two cold pain trials. Heart rate and blood pressure were assessed at baseline and after completion of the pain tasks.

RESULTS: Pain tolerance and pain intensity did not differ between children with chronic pain and controls for the unspecified trials. For the specified cold pressor trial, $92 \%$ of children with chronic pain completed the entire trial compared with only $61.5 \%$ of controls. Children with chronic pain exhibited a trend toward higher baseline and postsession heart rate and reported more anxiety and depression symptoms compared with control children.

CONCLUSIONS: Contextual factors related to the fixed trial may have exerted a greater influence on pain tolerance in children with chronic pain relative to controls. Children with chronic pain demonstrated a tendency toward increased arousal in anticipation of and following pain induction compared with controls.

Key Words: Acute pain; Cold pressor task; Laboratory pain; Pain intensity; Pressure pain; Thermal heat pain

$\mathrm{T}$ he question of whether children with chronic pain conditions show heightened pain responsivity compared with those without chronic pain is vexing. To address this question, experimental pain procedures may be used to control for potentially confounding variables inherent to clinical pain episodes such as variations in pain intensity and/or duration. The use of standardized, novel pain tasks also controls for an individual's previous pain history - a particular problem when examining 'real world' medical procedures. Nevertheless, evidence from existing laboratory studies about the responses of children with chronic pain relative to healthy children is mixed. Results have varied depending on the type of pain stimulation, the outcome measures as well as the patient population being tested. For example, several studies have found that children with chronic pain exhibit lower pressure pain thresholds relative to healthy controls. These findings have been reported in children with recurrent abdominal pain (RAP) $(1,2)$, sickle cell disease (3), juvenile idiopathic arthritis (JIA) $(4,5)$ and fibromyalgia (6). Lower rectal pain thresholds have also been reported in children with irritable bowel syndrome (7-9) and RAP $(7,8)$ compared with controls. However, recent investigations by Hermann et al (10) and Zohsel et al (11) have found no differences between children with RAP and controls in mechanical pain or heat

\author{
Les réponses expérimentales à la douleur chez les \\ enfants souffrant de douleur chronique et les \\ enfants en santé : comment diffèrent-elles?
}

HISTORIQUE : Des recherches étendues comparant les réponses à la douleur en laboratoire des enfants souffrant de douleur chronique à celles de sujets témoins en santé sont mitigées, certaines études indiquant une sensibilité à la douleur plus faible chez les sujets témoins et d'autres ne démontrant aucune différence. Peu d'études contiennent diverses modalités de douleur ou divers protocoles d'évaluation.

OBJECTIFS : Comparer les réponses à la douleur de 26 enfants (dont 18 filles) souffrant de douleur chronique et des sujets témoins appariés (âge moyen de 14,8 ans) à des tâches de laboratoire comportant une chaleur thermique, de la pression et une douleur par le froid. Les chercheurs ont évalué les réponses à la douleur par le froid au moyen de deux protocoles différents : un essai initial de durée indéterminée et un deuxième essai de durée déterminée.

MÉTHODOLOGIE : Les chercheurs ont effectué quatre essais de stimuli de douleur par pression et de douleur par chaleur thermique, tous de durée indéterminée, de même que les deux essais de douleur par le froid. Ils ont évalué la fréquence cardiaque et la tension artérielle avant puis après les tâches de douleur.

RÉSULTATS : La tolérance à la douleur et l'intensité de la douleur ne différaient pas entre les enfants ayant des douleurs chroniques et les sujets témoins lors des essais de durée indéterminée. Lors de l'essai de douleur par le froid à durée déterminée, $92 \%$ des enfants souffrant de douleur chronique ont terminé l'essai, par rapport à seulement $61,5 \%$ des sujets témoins. Les enfants souffrant de douleur chronique démontraient une tendance vers une fréquence cardiaque plus élevée avant et après la séance et déclaraient plus de symptômes d'anxiété et de dépression que les sujets témoins.

CONCLUSIONS : Des facteurs contextuels liés aux essais à durée déterminée peuvent exercer une plus grande influence sur la tolérance à la douleur chez les enfants souffrant de douleur chronique que chez les sujets témoins. Par rapport aux sujets témoins, les enfants souffrant de douleur chronique démontraient une tendance à une réaction d'alerte accrue en anticipation à l'induction de la douleur et après cette induction.

pain thresholds. These authors also found no differences in heat pain threshold between children with migraines compared with controls, although migraineurs showed lower mechanical pain thresholds than controls (12).

Whereas pain threshold is generally considered to be influenced primarily by sensory aspects of the pain experience, it is recognized that pain tolerance and pain intensity ratings may be influenced by a wide range of contextual, psychosocial factors including coping style, participant expectations and observer presence/behaviours (13). In two studies comparing children with JIA with controls on the cold pressor task $(14,15)$, there were no differences in pain threshold, but children with JIA exhibited lower pain tolerance than controls. In contrast, an analysis comparing healthy children with a clinical group comprised of children with RAP and children with anxiety disorders (16) found no differences in tolerance or pain intensity for the cold pressor task, although the clinical group exhibited higher heart rate at baseline and during the pain task. Although others have reported similar findings of increased physiological responses to the cold pressor task among children with RAP compared with controls (17-19), at least one study found no differences in blood pressure or heart rate among RAP children and control children in relation to the cold pressor task 
(20). Moreover, existing studies (all using the cold pressor task) have found no differences in pain intensity ratings between healthy controls and children with recurrent headaches (21), RAP (20) and JIA $(14,15)$.

As suggested by Hermann et al (10), the inconsistency of extant findings may be due to modality-specific alterations in pain sensitivity, patient heterogeneity or variations in testing protocols. In light of such possibilities, it is surprising that relatively few investigations have included more than one pain modality or utilized different types of pain assessment protocols within the same study. Zohsel et al (11) tested both pain threshold (discussed above) as well as "perceptual sensitization", defined as the increase in self-reported pain intensity following tonic or repetitive noxious stimulation to both heat and mechanical pain in children with chronic pain and controls. They found that children with RAP showed less perceptual sensitization in response to repetitive mechanical and tonic heat stimulation compared with controls (11). In a similar investigation comparing children with migraine with controls, Zohsel et al (12) found no group differences in perceptual sensitization when children were tested alone, but more perceptual sensitization among migraineurs to tonic heat stimulation when their mother was present.

Given the paucity of research testing multiple pain modalities and different pain assessment protocols within the same investigation, the present study sought to compare experimental pain responses in a group of children with chronic pain and a group of matched healthy control children across a variety of pain tasks involving different pain stimuli (ie, cold, thermal heat and pressure). We also sought to compare responses to two successive trials of the cold pressor task utilizing different experimental protocols. The first trial was open trial to assess pain tolerance; this trial functioned as a 'pure' test of cold pain tolerance because it asked participants to endure pain stimulation for as long as possible. The second trial consisted of a fixed 1 min ceiling in which participants were explicitly instructed to endure pain stimulation for $1 \mathrm{~min}$. The purpose of the fixed trial was to compare pain intensity ratings when cold water exposure was standardized across groups.

\section{METHODS}

\section{Participants}

Participants were 26 children with chronic pain and 26 control children; there were 18 girls (69.2\%) in each group. Because the children with chronic pain were all patients recruited from a chronic pain clinic (discussed below), they are referred to as the 'clinic sample'. Controls are also referred to as the 'community sample' because they were drawn from a larger sample of children recruited from the community $(n=192)$. The clinic and community samples were matched on age, sex and race/ethnicity. The decision to match the groups on race/ethnicity was based on the authors' previous work indicating racial/ethnic differences in children's laboratory pain responses and clinical pain characteristics $(22,23)$. Children ranged in age from eight to 18 years. The mean $( \pm \mathrm{SD})$ age for the clinic and the community samples was $14.8 \pm 2.2$ and $14.8 \pm 2.1$ years, respectively. There were equivalent numbers of Caucasian $(n=18 ; 69.2 \%)$ and African-American $(n=3$; $11.5 \%)$ participants in each group. There was one Latino $(3.8 \%)$ and four multiracial participants in the clinic group (14.5\%). For these clinic participants, controls of the same age and sex who were also of the same racial/ethnic background were sought, but no such matches were found. Thus, one mixed Caucasian-Asian participant in the clinic group was matched with an Asian participant in the community sample; the one Latino and three mixed Caucasian-Latino participants in the clinic group were matched with four Latino participants in the community sample. The clinic and the community groups did not differ with respect to socioeconomic status as measured by the Hollingshead scale (24), a composite score of parents' education and occupation (con-

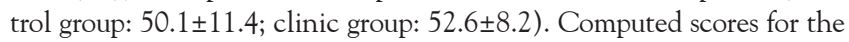
Hollingshead scale ranged from a high of 66 to a low of 6 , with higher numbers indicating higher socioeconomic status.
The clinic group consisted of patients presenting for treatment at a multidisciplinary, tertiary clinic specializing in pediatric chronic pain. Presenting pain diagnoses were: $42.3 \%$ headaches (migraines; myofascial, vascular, tension, stress-related or other type of headaches); $34.6 \%$ functional neurovisceral pain disorder (functional bowel, uterine or bladder disorder); $26.9 \%$ complex regional pain syndrome, type 1 or type $2,23.1 \%$ myofascial pain (of any part of the body excluding headaches); $19.2 \%$ fibromyalgia; and $7.7 \%$ joint pain (percentages sum to more than $100 \%$ due to multiple pain diagnoses). Multiple pain diagnoses were present in $38.5 \%(n=10)$ of the sample. Pain duration was between three months and one year for $44 \%$ of the sample, between one and four years for $31 \%$ of the sample, and four or more years for $20 \%$ of the sample.

A total of 80 clinic patients were approached about the study: 26 agreed to participate; seven were excluded due to medical conditions; nine declined to participate due to distance (many patients who attend the clinic live outside the greater Los Angeles [California, USA] area); 25 refused due to lack of time or interest; one refused due to anxiety about participation; and 12 expressed initial interest but subsequently could not be reached by telephone for screening/scheduling. Community sample participants were recruited by mass mailing, posted advertisements and classroom presentations. Potential participants were initially screened via telephone by a trained research assistant to determine study eligibility. Community sample participants were included if, by parent report, they did not have an acute or chronic illness (ie, heart condition or arthritis), recent injury or surgery on a limb, or history of frostbite or fainting spells. Parents were asked whether their child met any of the following exclusionary criteria: acute or chronic illness (as defined above); developmental delay or significant cognitive impairment that would preclude understanding of study procedures (eg, developmental age of less than eight years), or participation in pain induction procedures (eg, arm immersion in cold water); or daily use of opioid medication. After verbal consent was obtained from a parent, informed parent consent and child assent forms were mailed for review and signature. The Institutional Review Board of the University of California, Los Angeles, approved all study procedures.

\section{Procedure}

The procedure for the experimental pain study has been described in detail previously (25). Briefly, children completed questionnaires administered by an experimenter in a quiet room (only those questionnaires relevant to the current study are reported herein). Participants were then escorted into the laboratory where baseline heart rate and blood pressure were assessed using a digital blood pressure monitor. Participants then completed three laboratory pain tasks (pressure, thermal heat and cold pressor; described below) in counterbalanced order. Before each pain task, participants were informed that they would experience moderate sensation, which may eventually be perceived as pain. Participants were instructed to continue with each trial for as long as they could, but that they could end the trial if it became too uncomfortable and/or painful. At the end of the session, heart rate and blood pressure were reassessed after a $5 \mathrm{~min}$ rest period. Participants received a $\$ 30$ video store gift certificate and a T-shirt for their participation.

Medication use was assessed before the start of the laboratory session. All participants were previously instructed to abstain from opioid medication on the day of the laboratory session. None of the community sample participants reported any medication use. Most of the clinic sample participants $(n=21)$ reported using some type of medication that day; the most common medications were selective serotonin reuptake inhibitors $(n=12)$ and antiseizure medications $(n=8)$. Only one clinic participant reported using opioid medication; the results did not differ regardless of whether this participant was included or excluded from the analyses. The results reported below, therefore, include this participant.

Cold pressor task: For the cold pressor task (CPT), an ice chest filled with water maintained at $10^{\circ} \mathrm{C}$ was used; a plastic mesh screen 
separated crushed ice from a plastic armrest upon which the participant placed his or her hand. Two trials were conducted. In the first, participants placed their nondominant hand in the water for as long as they could. This open trial had a 3 min ceiling of which participants were not aware. Participants were told: "I'm going to ask you to put one hand in the water and keep it in for as long as you possibly can. When you can no longer keep your hand in, take it out. I may tell you to take out before you decide to do so. Try to keep it in as long as you possibly can."

The second trial consisted of a fixed 1 min ceiling using the dominant hand. Participants were told: "This time I'm going to ask you to put your other hand in the cold water. I'm going to ask you to leave your hand in for $1 \mathrm{~min}$. I will tell you when the time is up. When the time is up, you will take your hand out of the water. Try to keep your hand in until I tell you to take it out. But, if you need to, you can take it out before I tell you to."

Pressure task: The Ugo Basile Analgesy-Meter 37215 (Ugo Basile Biological Research Apparatus, Italy) administered focal pressure via a lucite point approximately $1.5 \mathrm{~mm}$ in diameter to the second dorsal phalanx of the middle finger and index finger of each hand. Four trials were conducted - two trials at each of two levels of pressure $(322.5 \mathrm{~g}$ and $465 \mathrm{~g}$ ). Each trial had an open 3 min ceiling. Participants were told: "During this task you will experience pressure on your finger. When I ask you to, you will place your finger onto this flat surface and I will lower this point onto your finger. The point should contact your finger at the spot I marked earlier. Leave your finger in place for as long as you can but you are free to remove the weight at any time. To remove the weight, lift this lever up like so."

Thermal heat task: The Ugo Basile 7360 Unit (Ugo Basile Biological Research Apparatus, Italy) administered four trials of radiant heat $5 \mathrm{~cm}$ proximal to the wrist and $7.6 \mathrm{~cm}$ distal to the elbow on both volar forearms. Two trials at each of two infrared stimulus intensities were conducted; each trial had an open 20 s ceiling. Participants were told: "This part of the experiment will involve heat. When you are ready, you will place your forearm over a small spot on this metal block. This spot will heat up quickly and will cause some discomfort. Keep your arm on the spot as long as you can, but you are free to remove your arm at any time."

\section{Measures}

Questionnaires: The Multidimensional Anxiety Scale for Children (MASC) (26) is a 39-item measure of anxiety symptoms with four subscales representing empirically derived domains of anxiety: physical symptoms, social anxiety, harm avoidance and separation anxiety. Items are scored on a four-point scale (never, almost never, sometimes, often); total and subscale scores are calculated by summing the relevant items, with higher scores indicating more anxiety. The MASC has shown high internal consistency (alpha $=0.88$ for total score) and adequate test-retest reliability (26). Scores range from 0 to 117 . T-scores (standardized to a mean $[ \pm \mathrm{SD}]$ of $50 \pm 10$ ) above 65 indicate possible clinical anxiety (26).

The Children's Depression Inventory (CDI) (27) is a 27-item instrument that assesses symptoms of depression, ranging from 0 (absence of symptom) to 2 (definite symptom). The CDI has demonstrated good internal consistency, adequate test-retest reliability, and validity. Scores range from 0 to 54; a cut-off of 12 has been used to indicate possible clinical depression (27).

The Child Health Questionnaire, Parent Report (CHQ; 28) is designed to assess parents' measurement of their child's physical and psychosocial well-being. The CHQ parent form is comprised of a number of subscales. For the purposes of assessing children's pain, the bodily pain subscale was the focus. The bodily pain subscale asks about intensity and frequency of children's overall bodily pain and discomfort. Higher scores indicate better functioning, ie, more freedom from pain. The measure has acceptable reliability and validity; it has been extensively tested in normative and clinical samples of children (28). Laboratory measures: Anxiety, pain intensity and pain bother ratings were measured using a vertical sliding visual analogue scale (VAS) anchored with 0 at the bottom indicating the least amount and 10 at the top indicating the greatest amount to rate anxiety, pain intensity, and pain bother. The scale had colour cues, graded from white at the bottom to dark red at the top, as well as a neutral face at the bottom and a negative facial expression at the top. Baseline anxiety ratings were assessed at the beginning of the laboratory session before the start of the pain tasks and anticipatory anxiety was assessed immediately before each trial. Pain intensity and pain bother ratings were assessed immediately after the end of each trial.

Pain tolerance: The time (in seconds) that elapsed from the onset of the pain stimulus to participants' withdrawal from the stimulus was recorded.

\section{Statistical analysis}

\section{RESULTS}

All VAS ratings and the tolerance data for the thermal heat and pressure tasks were highly correlated across the four trials ( $\mathrm{r}$ ranged from 0.55 to $0.97[\mathrm{P}<0.01])$ for both the clinic and control groups; these data were averaged across trials. Order effects for the pain tasks were tested (in the clinic and control groups separately), but none were found. The data were examined for violation of assumptions and outliers. Tolerance data for both trials of the CPT were bimodally distributed in both the clinic and control groups. As noted in a comprehensive review of the use of CPT in children (13), tolerance data are typically bimodally distributed. The CPT tolerance data were categorized into high versus low tolerance groups for the open trial and into completers versus noncompleters for the fixed trial (see details below). Tolerance data for both CPT trials were missing for one participant in the clinic group. In addition, baseline heart rate data were missing for one participant in the pain group.

Analyses comparing the clinic and control groups on the VAS data (all VAS ratings), the tolerance data for the thermal heat and pressure tasks, as well as baseline and postsession heart rate and blood pressure were conducted using a series of paired-samples $t$ tests. For the CPT tolerance data, McNemar's tests for related samples were conducted to determine whether membership in the high versus low tolerance and completer versus noncompleter groups differed between the clinic and control participants. A Bonferroni correction for the two main classes of pain response data (VAS ratings and tolerance) was used to reduce the likelihood of type 1 error. Therefore, a corrected alpha level of 0.025 (two-tailed) was used to evaluate the results. A trend was accepted for $0.025<\mathrm{P}<0.05$.

\section{Comparison of clinic versus control participants}

Table 1 summarizes descriptive data for the clinic and the control groups, as well as the results of the paired-samples $t$ tests. As shown in the table, the control group reported significantly less pain than the clinic group, as would be expected. The mean bodily pain score for the control group was comparable with that reported in a normative sample of children in the United States $($ mean $=81.7)$ and did not differ statistically according to a one-sample $t$ test. However, compared with controls, the clinic group reported more anxiety symptoms and more depressive symptoms. Examination of MASC scores indicated that mean scores for both the pain group (T-score mean $=52.4)$ and the control group $(\mathrm{T}$-score mean $=45.6) \mathrm{did}$ not indicate possible clinical anxiety as indexed by a T-score greater than 65 . Nevertheless, four children (15.4\%) in the clinic sample had MASC T-scores of 65 and above (versus no children in the community sample). The mean CDI score for the control group was less than 12 , whereas the mean CDI score for the clinic sample was greater than 12 - a cut-off that has been used to indicate possible clinical depression (27). More than one-half $(n=14 ; 53.8 \%)$ of the clinic sample scored at or above the CDI cut-off whereas only four $(15.4 \%)$ children in the community sample did. There was a trend toward group differences in baseline and postsession heart rate, with the clinic group exhibiting higher heart rate at both time points. There were no group differences in baseline anxiety, 


\section{TABLE 1}

Descriptive data and the results of the paired $t$ tests comparing the clinic and control groups

\begin{tabular}{|c|c|c|c|c|}
\hline & Clinic & Control & $t$ & $\mathbf{P}$ \\
\hline Bodily pain (CHQ) & $21.5 \pm 28.5$ & $79.2 \pm 14.4$ & 9.35 & 0.000 \\
\hline Anxiety symptoms (MASC) & $46.2 \pm 14.3$ & $36.3 \pm 11.0$ & -2.71 & 0.012 \\
\hline Depressive symptoms (CDI) & $12.5 \pm 7.5$ & $7.0 \pm 4.8$ & -3.93 & 0.001 \\
\hline Baseline anxiety (VAS) & $1.6 \pm 2.1$ & $1.7 \pm 2.4$ & 0.18 & 0.863 \\
\hline Baseline heart rate, beats/min & $75.9 \pm 11.6$ & $68.9 \pm 9.2$ & -2.12 & 0.045 \\
\hline \multicolumn{5}{|l|}{ Baseline blood pressure, $\mathrm{mmHg}$} \\
\hline Systolic & $107.0 \pm 12.2$ & $103.0 \pm 10.2$ & -1.31 & 0.203 \\
\hline Diastolic & $64.7 \pm 10.1$ & $61.9 \pm 8.3$ & -1.12 & 0.273 \\
\hline \multicolumn{5}{|l|}{ Pain intensity (VAS) } \\
\hline Thermal heat & $4.7 \pm 2.4$ & $4.1 \pm 2.5$ & -0.89 & 0.380 \\
\hline Pressure & $5.3 \pm 2.4$ & $4.0 \pm 2.7$ & -1.59 & 0.125 \\
\hline Cold pressor open & $3.4 \pm 2.8$ & $3.2 \pm 2.8$ & -0.17 & 0.867 \\
\hline Cold pressor fixed & $4.4 \pm 3.6$ & $3.6 \pm 3.1$ & -0.85 & 0.403 \\
\hline \multicolumn{5}{|l|}{ Pain bother (VAS) } \\
\hline Thermal heat & $4.0 \pm 3.0$ & $3.7 \pm 2.3$ & -0.48 & 0.634 \\
\hline Pressure & $4.0 \pm 2.6$ & $3.7 \pm 2.4$ & -0.39 & 0.703 \\
\hline Cold pressor open & $3.7 \pm 3.1$ & $3.4 \pm 2.6$ & -0.37 & 0.712 \\
\hline Cold pressor fixed & $4.3 \pm 3.9$ & $3.7 \pm 3.0$ & -0.51 & 0.612 \\
\hline \multicolumn{5}{|l|}{ Pain tolerance, $\mathrm{s}$} \\
\hline Thermal heat & $14.3 \pm 4.3$ & $13.7 \pm 5.4$ & -0.45 & 0.657 \\
\hline Pressure & $76.3 \pm 69.7$ & $56.9 \pm 58.3$ & -1.20 & 0.243 \\
\hline Cold pressor open (high tolerance) & $180.0 \pm 0.1$ & $179.7 \pm 0.3$ & N/A & A N/A \\
\hline Cold pressor open (low tolerance) & $25.4 \pm 16.0$ & $24.8 \pm 11.9$ & $\mathrm{~N} / \mathrm{A}$ & A N/A \\
\hline Cold pressor fixed (noncompleters) & $29.5 \pm 12.2$ & $26.0 \pm 10.3$ & N/A & A N/A \\
\hline Postsession heart rate, beats/min & $73.1 \pm 16.0$ & $65.8 \pm 8.5$ & -2.19 & 0.040 \\
\hline \multicolumn{5}{|l|}{ Postsession blood pressure, $\mathrm{mmHg}$} \\
\hline Systolic & $109.7 \pm 13.0$ & $104.9 \pm 10.0$ & -1.38 & 0.184 \\
\hline Diastolic & $64.6 \pm 9.7$ & $63.8 \pm 6.2$ & -0.69 & 0.500 \\
\hline
\end{tabular}

Data presented as mean $\pm S D$ unless otherwise indicated. CDI Children's Depression Inventory; CHQ Child Health Questionnaire; Cold pressor open Open trial; Cold pressor fixed Fixed 1 min trial; Cold pressor open (high tolerance) High tolerance participants; Cold pressor open (low tolerance) Low tolerance participants; Cold pressor fixed (noncompleters) Participants who did not complete the fixed trial; MASC Multidimensional Anxiety Scale for Children; N/A Not applicable (paired t tests cannot be conducted without fully matched pairs); VAS Visual analogue scale (0-10; $0=$ none, $10=$ extremely)

baseline blood pressure, VAS ratings for the open trials of pressure, thermal heat and cold pain, or post-session blood pressure. Tolerance for the thermal heat and pressure tasks did not differ between groups.

As noted above, the CPT tolerance data for both trials were bimodally distributed. Figure 1 illustrates the distribution of the tolerance times for the clinic and control groups. Inspection of the distributions indicated that for the first open trial, $62.7 \%$ of the total sample tolerated the cold water for $49 \mathrm{~s}$ or less (low tolerance group) and $37.3 \%$ were able to tolerate the entire $3 \mathrm{~min}$ trial (high tolerance group). Membership in the high versus low tolerance group for the open CPT trial did not differ between the clinic and control groups (Figure 1). Inspection of the distributions for the total sample for the fixed CPT trial indicated that $23.5 \%$ were not able to tolerate the trial for the entire minute (noncompleters; mean tolerance 26.6 $\pm 10.1 \mathrm{~s}$ ) whereas $76.5 \%$ were able to complete the entire trial (completers). The proportion of completers versus noncompleters for the fixed trial differed significantly between the clinic and control groups (McNemar's $\left.\chi^{2}(1)=6.57[\mathrm{P}<0.001]\right)$. Power analysis indicated that power for a twosided test with 26 matched pairs for a McNemar's test of correlated proportions was 0.95, with alpha set at 0.025. As illustrated in Figure 1, all but two participants in the clinic group (92\%) were able to complete the entire trial whereas the control group was more evenly split

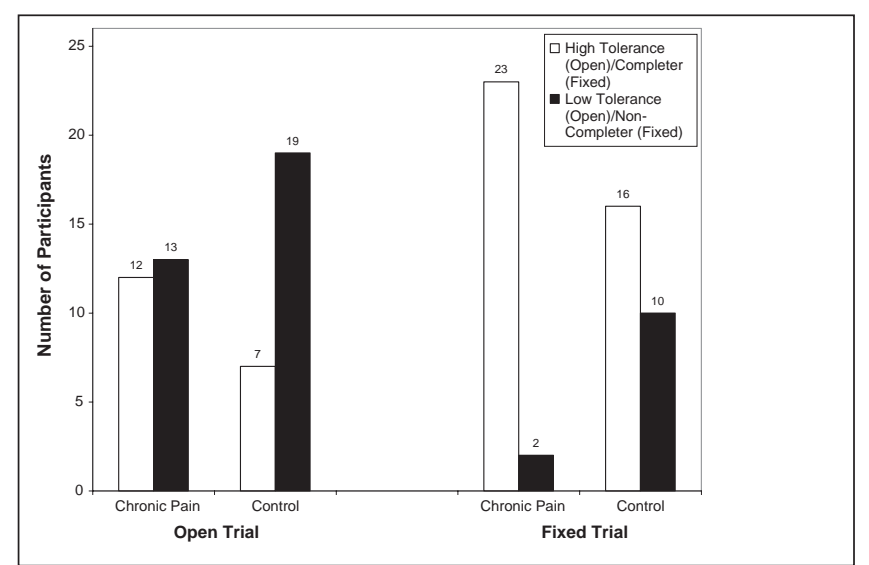

Figure 1) Distribution of tolerance data for the cold pressor trials according to group

between completers $(61.5 \%)$ and noncompleters (38.5\%). Pain ratings (both intensity and bother) for the fixed trial did not differ between the clinic and control groups. These results also held when pain ratings for completers only were tested. As shown in Table 1, the mean tolerance times for noncompleters were similar across the clinic and control groups.

\section{DISCUSSION}

The current study compared the pain responses of children with chronic pain and matched controls from the community to standardized pain tasks involving thermal heat, pressure and cold pain stimuli. Our results indicated that children with chronic pain did not differ from controls in pain intensity or pain tolerance across all three pain modalities when exposed to open-ceiling trials. However, surprisingly, children with chronic pain were significantly more likely to tolerate a fixed 1 min cold pressor trial compared with controls (Figure 1). All but two children with chronic pain $(92 \%)$ were able to complete the trial compared with only $61.5 \%(n=16)$ of control children. The findings for the fixed trial are even more surprising given that for the open cold pressor trial, children with chronic pain and control children were equally likely to belong to the low tolerance group (ie, tolerance times of $49 \mathrm{~s}$ or less). Despite differences in pain tolerance for the fixed trial, pain intensity ratings did not differ between children with chronic pain and controls, and mean tolerance times for noncompleters did not differ. Additional findings indicated that children with chronic pain exhibited a trend toward higher heart rate at baseline, before the start of the laboratory pain tasks, and after the pain tasks were completed, relative to controls (Table 1). Neither blood pressure nor ratings of anxiety differed between groups at baseline. However, children with chronic pain reported more anxiety and depressive symptoms than controls.

Overall, our findings suggest that children with chronic pain do not exhibit heightened pain responsivity compared with their healthy counterparts when exposed to a variety of pain modalities during 'pure' assessments of pain tolerance (ie, open ceiling trials). These results are consistent with other investigations reporting no differences in pain threshold $(10,11)$, pain tolerance $(16)$ or pain intensity ratings $(14-16,20,21)$ between these groups of children. Our results partly agree with those of Zohsel et al (12), who found no differences in heat pain threshold between children with chronic pain and controls, but did find lower mechanical pain thresholds among children with chronic pain. However, our findings conflict with studies reporting lower pressure pain thresholds $(1,2,4-9,29)$ and lower cold pain tolerance $(14,15)$ for children with chronic pain compared with controls. Differences in assessment protocol (eg, stimulus intensity) or sample characteristics (eg, age range) may account for the divergent findings. For example, previous studies have found lower cold pain tolerance for children with JIA relative to controls $(14,15)$, but our sample of 
children with chronic pain did not include any participants with JIA. We did not assess pain threshold in the current study; previous work in our laboratory has indicated that some children naturally use distraction to cope with pain and that instructions to attend to pain sensation necessitated by the assessment of pain threshold may interfere with the use of distraction, thereby altering the ability to tolerate pain (30). Thus, studies on pain threshold in children that do not control for natural coping style during pain exposure may introduce confounds that complicate the interpretation of results. For these reasons, we chose to assess pain intensity and pain tolerance times, rather than pain threshold.

Our results for the fixed trial are consistent with those of Zohsel et al (11), who reported that children with RAP evidenced less perceptual sensitization than controls in response to repetitive mechanical and tonic heat stimulation. However, even though children with chronic pain exhibited greater tolerance than controls for the fixed trial in the present study, pain ratings for the fixed trial did not differ between groups. These findings suggest that factors other than sensory aspects of pain may have a greater impact on pain tolerance among children with chronic pain, relative to controls. Although we did not explicitly test which of these other contextual factors were at play, one possibility is that children with chronic pain have a greater need to act in ways that would be viewed favourably by others (ie, high social desirability). Thus, children with chronic pain may have wanted to please the experimenter by completing the entire trial. In one of the few studies examining social desirability among children with chronic pain, children with high social desirability scores reported fewer anxiety/depression symptoms compared with lower scorers, but no differences were reported for somatic symptoms, pain duration or pain-related disability (31). Nevertheless, a control group was not included in this work and, therefore, it is unknown whether children with chronic pain differ from healthy children in social desirability. A related explanation is that children with chronic pain may be more compliant with laboratory procedures given their history of undergoing frequent medical procedures for their pain. Earlier, we found that among such patients, the average number of medical visits for pain in the previous year was $6.26(32)$.

Another possibility is that pain tolerance among children with chronic pain may be especially influenced by the presence and/or behaviour of the experimenters during pain stimulation. Experimenters (who were aware of to which group each child belonged) may have sent subtle cues that differed between groups even though the protocol was standardized and experimenters were highly trained. Previous work has demonstrated the effect of parent presence/behaviours on children's experimental pain responsivity. Zohsel et al (12) found that children with migraines had similar heat pain thresholds to controls when tested alone, but migraineurs showed more perceptual sensitization to tonic heat stimulation when their mother was present. Walker et al (33) reported that compared with a no instruction control condition, symptom complaints by children with RAP and healthy children following induction of visceral discomfort increased when mothers devoted attention to their symptoms and were reduced when mothers distracted the children; the effect of mother attention on symptom complaints was greater for girls with RAP than for boys with RAP or healthy children (33). We were not able to test for sex differences in pain responsivity due to the small number of boys in the current study. However, the proportion of girls and boys among fixed trial noncompleters was similar across groups. For the chronic pain group, one boy (50\%) and one girl were noncompleters, and for the control group four boys $(40 \%)$ and six girls (60\%) were noncompleters.

An alternative explanation is that pain tolerance for the fixed trial was more heavily influenced by expectations engendered by the task instructions in children with chronic pain than controls. For instance, children with chronic pain may have interpreted the instructions as indicating that they should be able to tolerate the full trial. This desire to maintain stoicism in the face of pain is somewhat distinct from a more general need to appear 'good' in front of others. Previously, we found that attempts to hide pain from others was the most common theme from our narrative analysis of children's experience with chronic pain, reported by $62 \%$ of children (32). Another possibility is that informing children of the amount of time they would be exposed to pain may have differentially influenced responses. An earlier study involving adults found that time estimates for a cold pressor trial were more accurate among those given a time-specific goal for tolerance compared with those not given a time-specific goal (34). A specific time goal may have distracted subjects from their pain by focusing their attention instead on the passage of time. Given their experience with frequent pain episodes, it may be that children with chronic pain are more adept at using this type of distraction compared with healthy children. Relatedly, the fixed ceiling may have increased the perception of controllability and/or predictability of pain exposure, thereby decreasing its noxiousness among children with chronic pain. In adults, the desire for control and prediction of having control was associated with level of pain during dental treatment (35). Given that clinical pain episodes are often difficult to control and/or predict, the fixed trial in particular may have been comparatively 'easy' for children with chronic pain to endure.

Although we did not assess which, if any, of these cognitive factors may have influenced children in the current study, one clinical implication of our findings is that if such factors are confirmed to influence pain responses among children with chronic pain, it follows that efforts to actively change such cognitions may lead to changes in painrelated behaviour. This notion is a fundamental principle underlying cognitive-behavioural treatment approaches to chronic pain that seek to alter patients' cognitions about pain to affect behaviour change. An additional clinical implication of our results is that behavioural interventions may incorporate laboratory-based pain exposures to teach and reinforce changes in pain tolerance over time. Newly developed behavioural approaches to pediatric chronic pain have begun to include similar, task-based pain exposures (36). Giving children with chronic pain concrete evidence of increased pain tolerance during controlled pain exposures may lead to improvements in pain self-efficacy and a greater sense of mastery over pain.

Our finding that relative to controls, children with chronic pain exhibited a trend toward increased physiological arousal as indexed by higher heart rate in anticipation of and following pain induction is in line with previous investigations. One study found that a clinical group of children with RAP and anxiety disorders evidenced higher heart rate at baseline compared with controls; elevated heart rate was also reported in the clinical group during the CPT (16). Others have similarly found increased physiological responses following pain induction among children with RAP relative to control children (17-19). Our findings contradict one investigation in which children with RAP did not differ from controls in blood pressure or heart rate responses following the CPT (20). Despite exhibiting heightened physiological arousal before pain stimulation, children with chronic pain in the present study did not report increased anticipatory anxiety relative to control children. This result is somewhat surprising given that children with chronic pain reported more anxiety symptoms (as well as depressive symptoms) compared with controls - findings that have been commonly reported in the literature (37). On the other hand, we have previously found in a sample of healthy children that anxiety symptoms are not directly related to pain-related anticipatory anxiety (38).

Limitations to the present study should be noted. Children with chronic pain who agreed to undergo pain induction may differ from those who did not participate. It is possible that the current sample of children with chronic pain may have been less anxious or more compliant than those who refused participation. An important factor differentiating the chronic pain and control samples is that the former was recruited among patients from a tertiary chronic pain clinic whereas the latter was recruited in a different way from the wider community. Thus, group differences may have as much to do with selection factors such as parental concern, solicitude, fear of medical problems, 
or family resources and access to medical care, as with children's pain symptoms. As noted above, we did not include a measure of social desirability to assess the possible effects of this construct on pain responses. We also did not ask about children's thoughts and expectations regarding the pain tasks, and such qualitative data would have provided information on why children with chronic pain behaved differently from controls in relation to the fixed trial. As mentioned above, we were not able to test for sex differences due to the small number of boys. However, the sample composition was dictated by the higher proportion of girls in the chronic pain group - such sex differentials in a pediatric chronic pain sample have been previously reported $(32,39)$. The present study tested the effects of acute pain induction and the results may not hold for tasks designed to induce sensations that more closely reflect the experience of chronic pain. Finally, our assessment of physiological responses was limited to a single measure of heart rate and blood pressure at baseline and postsession; future studies may employ more sophisticated systems to continuously assess multiple indices of physiological response during pain induction.

Overall, our findings indicate no differences between healthy children and children with chronic pain when exposed to laboratory pain tasks with open ceilings. However, when exposed to a fixed trial of cold pain, children with chronic pain were more likely to complete the entire trial than were healthy children, despite reporting similar pain intensity ratings as the healthy control group. The chronic pain group also evidenced a trend toward higher baseline and postsession heart rates and endorsed more anxious and depressive symptoms than the control group, suggesting higher levels of general arousal and mood disturbances. Nevertheless, the lack of group differences in anticipatory anxiety suggest that the laboratory pain procedures in themselves were not additionally stressful for the children with chronic pain. Our findings indicate that children with chronic pain may be particularly sensitive to contextual factors such as social desirability, pain-related social expectations and perceptions of controllability/predictability engendered by specific types of pain induction. Future work may use qualitative data to explore relevant themes in relation to pain coping, perceived control and other salient factors. Such information, together with quantitative data on acute pain responses, may paint a more comprehensive picture of the unique and specific characteristics that influence the pain responses of children with chronic pain, and may inform the development of interventions that seek to alleviate pain in this population.

ACKNOWLEDGEMENTS: This study was supported by R01DE012754, awarded by the National Institute of Dental and Craniofacial Research (PI: Lonnie K Zeltzer), by UCLA General Clinical Research Center Grant M01-RR-00865 (PI: Lonnie K Zeltzer), and by 1K01AT005093, awarded by the National Center for Complementary and Alternative Medicine (PI: Subhadra Evans). There are no conflicts of interest in the research reported in this article.

\section{REFERENCES}

1. Alfven G. The pressure pain threshold (PPT) of certain muscles in children suffering from recurrent abdominal pain or nonorganic origin - an algometric study. Acta Paediatr 1993;82:481-3.

2. Duarte MA, Goulart EMA, Penna FJ. Pressure pain threshold in children with recurrent abdominal pain. J Pediatr Gastroenterol Nutr 2000;31:280-5.

3. Walco GA, Dampier CD, Hartstein G, Djordjevic D, Miller L. The relationship between recurrent clinical pain and pain threshold in children. In: Tyler DC, Krane EJ, eds. Advances in Pain Research Therapy. New York: Raven Press Ltd, 1990:333-40.

4. Hogeweg JA, Kuis W, Huygen ACJ, et al. The pain threshold in juvenile chronic arthritis. Br J Rheumatol 1995;34:61-7.

5. Hogeweg JA, Kuis W, Oostendorp RA, Helders PJ. General and segmental reduced pain thresholds in juvenile chronic arthritis. Pain 1995;62:11-7.

6. Buskila D, Press J, Gedalia A, et al. Assessment of nonarticular tenderness and prevalence of fibromyalgia in children. J Rheumatol 1993;20:368-70.

7. Di Lorenzo C, Youssef NN, Sigurdsson L, Scharff L, Griffiths J, Wald A. Visceral hyperalgesia in children with functional abdominal pain. J Pediatr 2001;139:838-43.

8. Faure C, Wieckowska A. Somatic referral of visceral sensations and rectal sensory threshold for pain in children with functional gastrointestinal disorders. J Pediatr 2007;150:66-71.

9. Van Ginkel R, Voskuijl WP, Benninga MA, Taminiau JA, Boeckxstaens GE. Alterations in rectal sensitivity and motility in childhood irritable bowel syndrome. Gastroenterology 2001;120:31-8.

10. Hermann C, Zohsel K, Hohmeister J, Flor H. Cortical correlates of an attentional bias to painful and innocuous somatic stimuli in children with recurrent abdominal pain. Pain. 2008;136:397-406.

11. Zohsel K, Hohmeister J, Flor H, Hermann C. Somatic pain sensitivity in children with recurrent abdominal pain. Am J Gastroenterol 2008;103:1517-23.

12. Zohsel K, Hohmeister J, Oelkers-Ax R, Flor H, Hermann C. Quantitative sensory testing in children with migraine: Preliminary evidence for enhanced sensitivity to painful stimuli especially in girls. Pain 2006;123:10-8.

13. von Baeyer CL, Piira T, Chambers CT, Trapanotto M, Zeltzer LK. Guidelines for the cold pressor task as an experimental pain stimulus for use with children. J Pain 2005;6:218-27.

14. Thastum M, Zachariae R, Herlin T. Pain experience and pain coping strategies in children with juvenile idiopathic arthritis. J Rheumatol 2001;28:1091-8.

15. Thastum M, Zachariae R, Scholer M, Bjerring P, Herlin T. Cold pressor pain: Comparing responses of juvenile arthritis patients and their parents. Scand J Rheumatol 1997;26:272-9.

16. Dufton LM, Dunn MJ, Slosky LS, Compas BE. Self-reported and laboratory-based responses to stress in children with recurrent pain and anxiety. J Pediatr Psychol 2011;36:95-105.

17. Apley J, Haslam DR, Tulloh CG. Pupillary reaction in children with recurrent abdominal pain. Arch Dis Child 1971;46:337-40.

18. Dorn LD, Thato S, DiLorenzo C, Chandra R, Dahl RD, Campo JV. A preliminary report of differences in physiologic reactivity in the cold pressor test by context in 8 to 16 year-olds: Home versus laboratory measurements. J Adolesc Health 2004;34:138.

19. Rubin LS, Barbero GJ, Sibinga MS. Pupillary reactivity in children with recurrent abdominal pain. Psychosom Med 1967;29:111-20.

20. Feuerstein M, Barr RG, Francoeur TE, Houle M, Rafman S. Potential biobehavioral mechanisms of recurrent abdominal pain in children. Pain 1982;13:287-98.

21. Smith MS, Martin-Herz SP, Womack WM, McMahon RJ. Recurrent headache in adolescents: Nonreferred versus clinic population. Headache 1999;39:616-24.

22. Evans S, Lu Q, Tsao JC, Zeltzer LK. The role of coping and race in healthy children's experimental pain responses. J Pain Manag 2008;1:151-62.

23. Evans S, Taub R, Tsao JC, Meldrum M, Zeltzer LK. Sociodemographic factors in a pediatric chronic pain clinic: The roles of age, sex and minority status in pain and health characteristics. J Pain Manag 2010;3:273-81.

24. Hollingshead AB. Four-Factor Index of Social Status. Yale University: Department of Sociology, 1975.

25. Tsao JC, Myers CD, Craske MG, Bursch B, Kim SC, Zeltzer LK. Role of anticipatory anxiety and anxiety sensitivity in children's and adolescents' laboratory pain responses. J Pediatr Psychol 2004;29:379-88.

26. March JS, Parker JD, Sullivan K, Stallings P, Conners CK. The Multidimensional Anxiety Scale for Children (MASC): Factor structure, reliability, and validity. J Am Acad Child Adolesc Psychiatry 1997;36:554-65.

27. Kovacs M. Children's Depression Inventory (CDI). New York: Multi-Health Systems, Inc, 1992.

28. Landgraf JL, Abetz L, Ware JE. The CHQ User's Manual, 2nd edn. Boston: HealthAct, 1999.

29. Chambers CT, Craig KD, Bennett SM. The impact of maternal behavior on children's pain experiences: An experimental analysis. J Pediatr Psychol 2002;27:293-301. 
30. Fanurik D, Zeltzer LK, Roberts MC, Blount RL. The relationship between children's coping styles and psychological interventions for cold pressor pain. Pain 1993;53:213-22.

31. Logan DE, Claar RL, Scharff L. Social desirability response bias and self-report of psychological distress in pediatric chronic pain patients. Pain 2008;136:366-72.

32. Meldrum ML, Tsao JC, Zeltzer LK. "I can't be what I want to be": Children's narratives of chronic pain experiences and treatment outcomes. Pain Med 2009;10:1018-34.

33. Walker L, Williams S, Smith C, Garber J, Van Slyke D, Lipani T. Parent attention versus distraction: Impact on symptom complaints by children with and without chronic functional abdominal pain. Pain 2006;122:43-52.

34. Thorn BE, Hansell PL. Goals for coping with pain mitigate time distortion. Am J Psychol 1993;106:211-25.
35. Gedney JJ, Logan H. Perceived control and negative affect predict expected and experienced acute clinical pain: A structural modeling analysis. Clin J Pain 2007;23:35-44.

36. Allen LB, Tsao JCI, Seidman LC, Ehrenreich-May J, Zeltzer LK. A unified, transdiagnostic treatment for adolescents with chronic pain and comorbid anxiety and depression. Cogn Behav Pract 2012;19:56-67.

37. Slover R, Neuenkirchen GL, Olamikan S, Kent S. Chronic pediatric pain. Adv Pediatr 2010;57:141-62.

38. Tsao JC, Lu Q, Kim SC, Zeltzer LK. Relationships among anxious symptomatology, anxiety sensitivity and laboratory pain responsivity in children. Cogn Behav Ther 2006;35:207-15.

39. Meldrum ML, Tsao JC, Zeltzer LK. "Just be in pain and just move on": Functioning limitations and strategies in the lives of children with chronic pain. J Pain Manag 2008;1:131-41. 


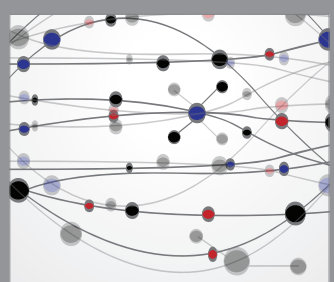

The Scientific World Journal
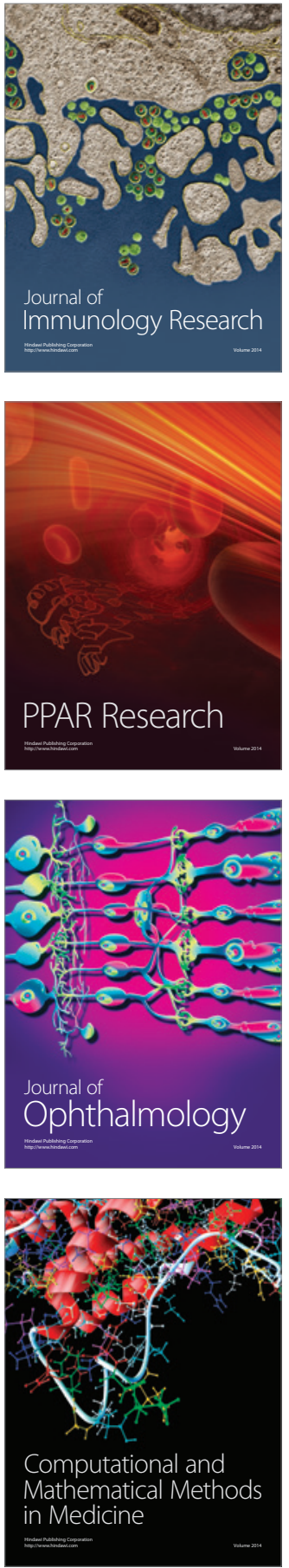

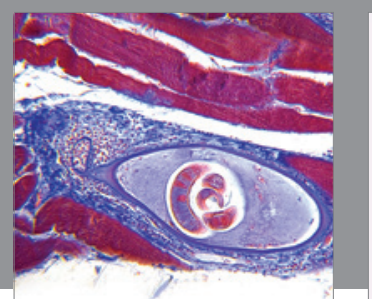

Gastroenterology Research and Practice

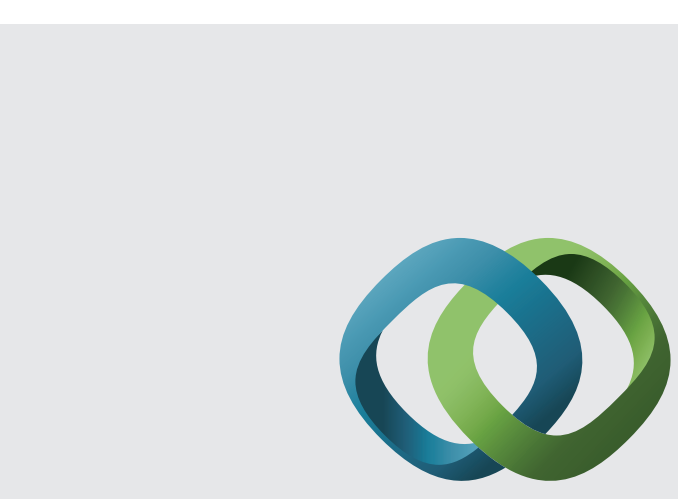

\section{Hindawi}

Submit your manuscripts at

http://www.hindawi.com
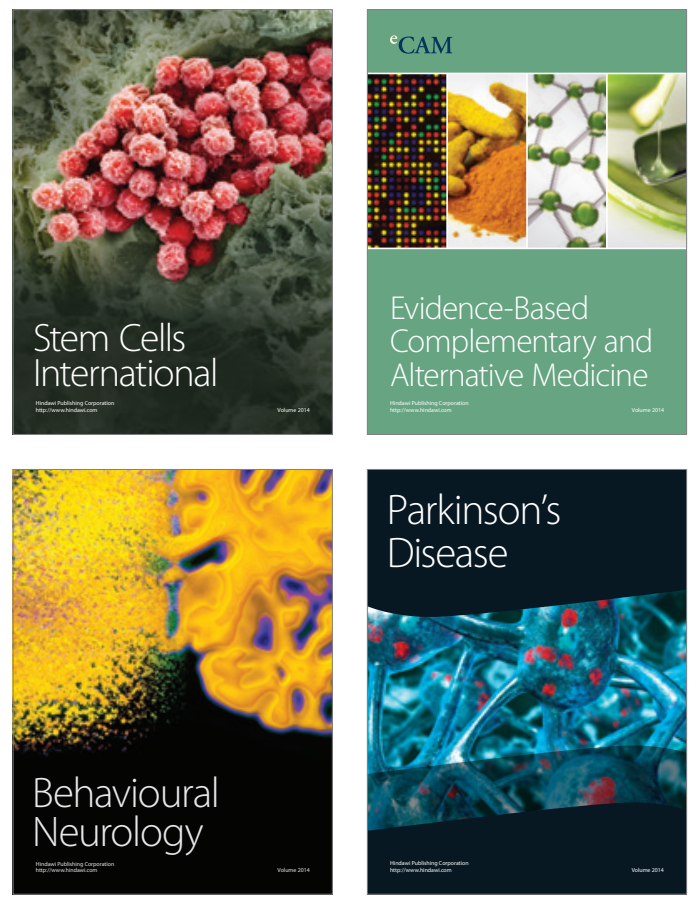
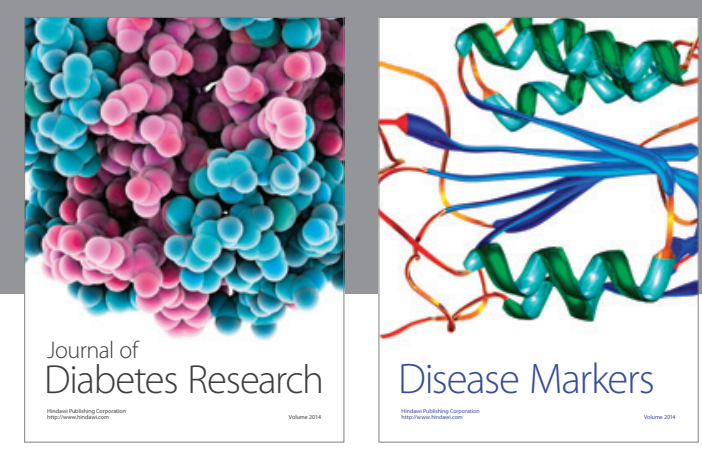

Disease Markers
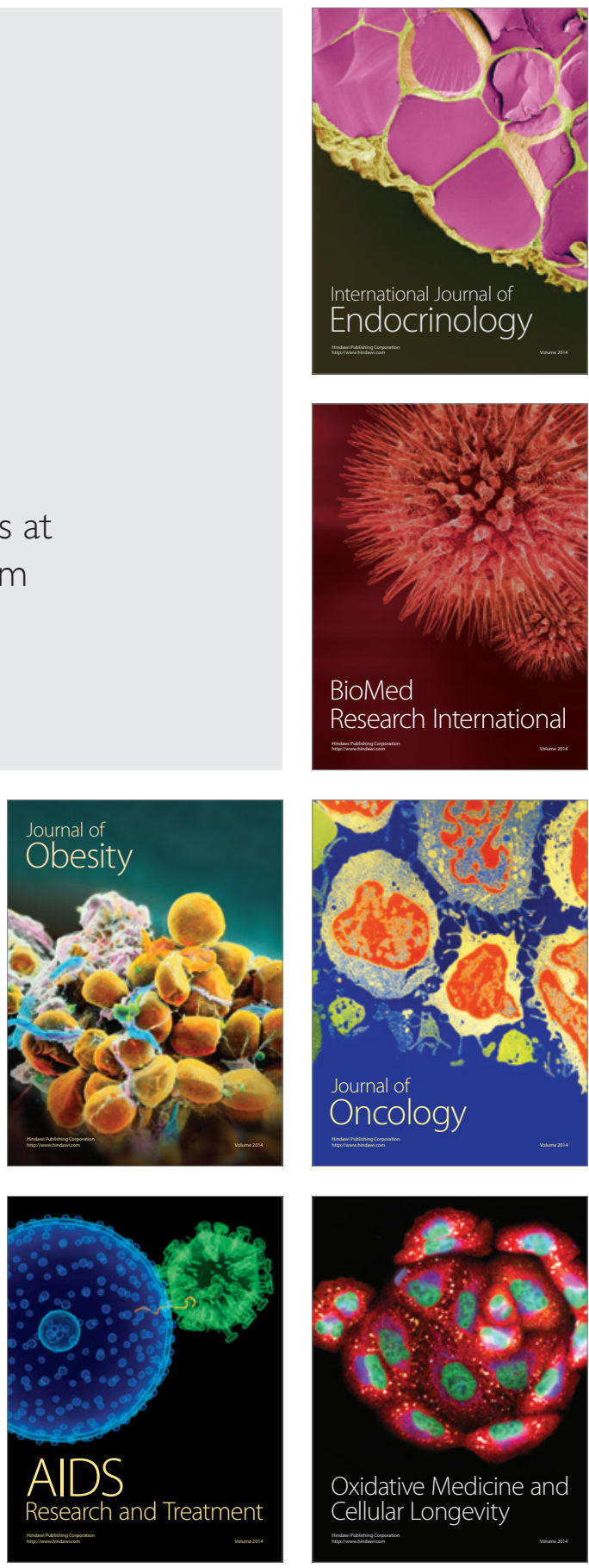